\title{
Existence of Unique Weak Solutions to a Dynamical System for Nonlinear Elastomers with Hysteresis *
}

\author{
H.T. Banks, Gabriella A. Pintér and Laura K. Potter \\ Center for Research in Scientific Computation \\ Department of Mathematics \\ North Carolina State University \\ Raleigh, NC 27695-8205
}

\begin{abstract}
We consider a class of dynamic models for elastomers involving nonlinear viscoelastic (hysteresis) as well as nonlinear finite elastic components of the constitutive laws. Existence and uniqueness results are presented along with sample numerical fits to experimental data to demonstrate the efficacy of the models.
\end{abstract}

*AMS Subject Classification: 34G20, (35L70, 47H30,73G05)

Research supported in part by the U. S. Air Force Office of Scientific Research under grants AFOSR F4962098-1-0180 and AFOSR F49620-98-1-0430 and in part by the National Science Foundation by an NSF-GRT Fellowship to Laura K. Potter under grant GER-9454175. 


\section{Introduction}

In this paper we present a well-posedness result for a new model describing the behavior of nonlinear elastomers. These models for filled (e.g., with carbon black) rubber-like elastomers are motivated by formulations for large deformation tensile dynamics. When properly modeled, these dynamics require finite strains [31, 33]

$$
\hat{e}=\frac{\partial u}{\partial x}+\frac{1}{2}\left(\frac{\partial u}{\partial x}\right)^{2}=\epsilon+\frac{1}{2} \epsilon^{2}
$$

in constitutive laws, where $\epsilon=\frac{\partial u}{\partial x}$ is the usual infinitesimal strain of linear elasticity (see $[8,14]$ for basic modeling techniques as well as specific examples related to longitudinal (tensile) deformation models). Computational and experimental results $[9,10,11,13]$ have confirmed that nonlinear constitutive laws

$$
\sigma(t)=\tilde{G}_{e}(\hat{e}(t))
$$

are adequate but required to model even the small deformation elastic dynamics for lightly filled elastomers. However, for more highly filled elastomers (the primary focus for both passive and active damping devices), hysteresis is present and viscoelastic behavior must be combined with basic nonlinear elastic behavior in constitutive laws of the form

$$
\sigma(t)=\tilde{G}_{e}(\hat{e}(t))+\tilde{G}_{v}(\hat{e}(t+s):-\infty<s \leq 0) .
$$

In light of (1.1), this is equivalent to attempting to determine nonlinear maps $\tilde{g}_{e}, g_{v}$ so that the constitutive law is given by

$$
\sigma(t)=\tilde{g}_{e}(\epsilon(t))+g_{v}(\epsilon(t+s):-\infty<s \leq 0)
$$

We choose to model this hysteresis using a Boltzmann type law of the form

$$
\sigma(t)=\tilde{g}_{e}(\epsilon(t))+\int_{-\infty}^{t} Y(t-s) \frac{d}{d s} g_{v}(\epsilon(s), \dot{\epsilon}(s)) d s,
$$

where $\sigma$ denotes the stress and $\epsilon$ the infinitesimal strain. This stress-strain law implies that the stress depends not only on the current strain but also on the history of the strain and the strain-rate. This law contains several standard internal strain or internal variable formulations as special cases. The ADF models of Lesieutre [24, 25] for composite materials exhibiting both elastic and anelastic displacement fields are formulated on the assumption 
that the host elastic material contains anelastic materials with internal strains $\epsilon_{1}$ which are elastic strain driven, i.e., the constitutive laws have the form

$$
\sigma(t)=E_{1} \epsilon(t)-E_{2} \epsilon_{1}(t)
$$

where the internal strain is described by

$$
\dot{\epsilon}_{1}(t)+c_{1} \epsilon_{1}(t)=c_{2} \epsilon(t)
$$

or

$$
\epsilon_{1}(t)=\int_{0}^{t} c_{2} e^{-c_{1}(t-s)} \epsilon(s) d s
$$

More generally, one might have a nonlinear version of (1.7) given by

$$
\dot{\epsilon}_{1}(t)+c_{1} \epsilon_{1}(t)=g_{v}(\epsilon(t)) \text {. }
$$

On the other hand, Johnson et. al., [22] propose a linear alternative to (1.7)

$$
\dot{\epsilon}_{1}(t)+c_{1} \epsilon_{1}(t)=c_{1} \dot{\epsilon}(t)
$$

in which the internal strain is strain rate driven, with nonlinear generalization given by

$$
\dot{\epsilon}_{1}(t)+c_{1} \epsilon_{1}(t)=g_{v}(\dot{\epsilon}(t)) \text {. }
$$

Our attempts to use models equivalent to (1.6) with either (1.8) or (1.10) to describe either quasi-static or dynamic tensile test data [13] have not been successful. However, more general models of the form (1.5) which correspond to an internal strain model of the form

$$
\dot{\epsilon}_{1}(t)+c_{1} \epsilon_{1}(t)=\frac{d}{d t} g_{v}(\epsilon(t), \dot{\epsilon}(t))
$$

have performed quite well in modeling our quasi-static and dynamic tensile experimental data. In these applications we required that the derivative $\frac{d}{d t}$ in (1.5) be a distributional derivative (in the sense described below) and that $g_{v}$ have the form

$$
g_{v}(\epsilon(s), \dot{\epsilon}(s))=\left\{\begin{array}{ll}
g_{v i}(\epsilon(s)) & \text { if } \dot{\epsilon}(s)>0 \\
g_{v d}(\epsilon(s)) & \text { if } \dot{\epsilon}(s)<0
\end{array}\right\}
$$

i.e., the viscoelastic response differs when the material is loading from that when it is unloading. This is one novel feature of our model which is based on our experimental findings [13]. In the following we shall refer to the nonlinearities $\tilde{g}_{e}$ and $g_{v}$ as the elastic and viscoelastic 
response functions, respectively. Our kernel function $Y$ is assumed positive, decreasing and is not singular at 0 . In practice, we consider $Y(\xi)=c_{2} \exp \left(-c_{1} \xi\right)$, where $c_{1}, c_{2}$ are positive. Moreover, we expect that the material does not exhibit infinite memory, but depends significantly only on history of finite length, $r$. Exponential memory kernels $Y$ are such that $Y(\xi) \approx 0$ for $\xi \geq r$, and $r$ sufficiently large. Therefore, we approximate (1.5) by

$$
\sigma(t)=\tilde{g}_{e}(\epsilon(t))+\int_{t-r}^{t} Y(t-s) \frac{d}{d s} g_{v}(\epsilon(s), \dot{\epsilon}(s)) d s .
$$

In a typical experiment the rubber rod loads and unloads periodically. Let us suppose that it loads from $t_{K}$ to $t_{K+1}$ when $K$ is even, where $t_{0}=0$.

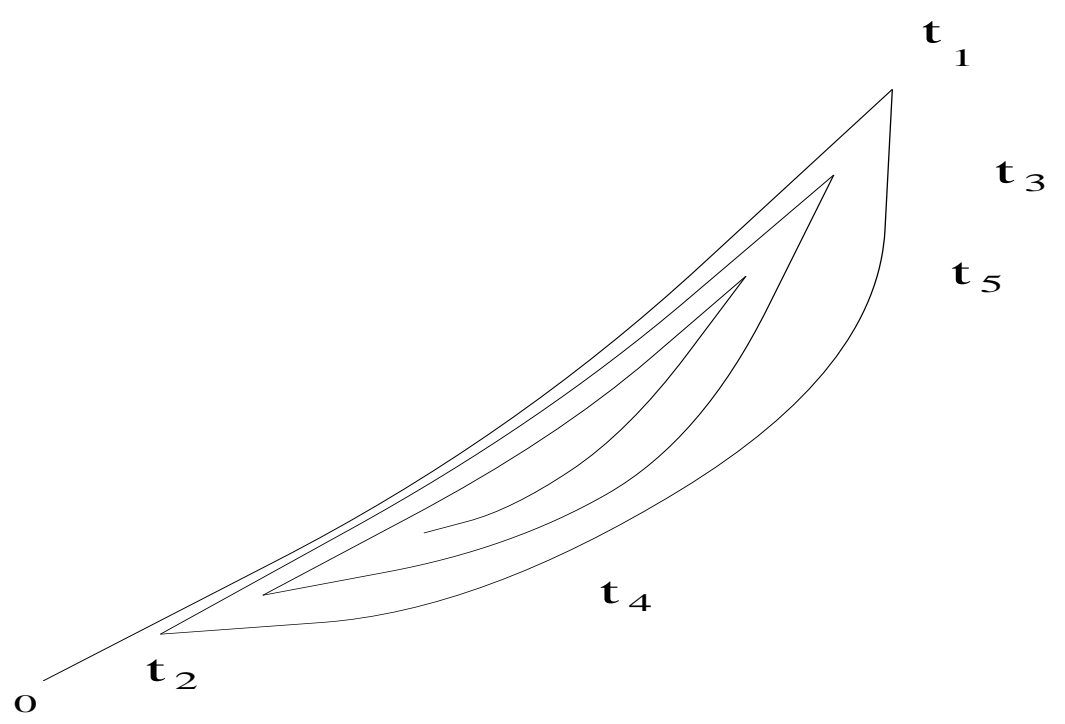

Figure 1: Loading and unloading of the rubber rod

Assuming that $t_{K}<t<t_{K+1}$, where $\mathrm{K}$ is odd, and $r>t_{\max }$, where $t_{\max }$ is the maximum value of $t$ of interest, we may integrate by parts in the integral term in (1.12) interval by interval to obtain:

$$
\begin{aligned}
& \sigma(t)=\tilde{g}_{e}(\epsilon(t))+Y(0) g_{v d}(\epsilon(t))+\int_{t-r}^{t} \dot{Y}(t-s) g_{v}(\epsilon(s)) d s \\
& +\sum_{k=1}^{K} Y\left(t-t_{k}\right)(-1)^{k+1}\left[g_{v i}\left(\epsilon\left(t_{k}\right)\right)-g_{v d}\left(\epsilon\left(t_{k}\right)\right)\right] .
\end{aligned}
$$

We shall refer to the terms in the summation as "jump terms" and the $\left\{t_{k}\right\}$ as "turning points". We note that we obtain a similar system in the case the rubber rod is loading, i.e., $t_{K}<t<t_{K+1}, \mathrm{~K}$ even. 
We consider the longitudinal motion of an elastomer rod of length $\ell$ and let $u(t, x)$ denote the displacement at time $t$ of the section of the rod originally located at $x, 0 \leq x \leq \ell$. The motion is governed by the equation (for a careful derivation see $[8,11]$ )

$$
\rho A_{c} u_{t t}=A_{c} \sigma_{x}(x, t)+f(x, t)
$$

where $A_{c}$ is the undeformed cross sectional area, and $\rho$ is the density. Combining our stress strain law with (1.14) we obtain the model for $t_{K}<t<t_{K+1}$, K odd:

$$
\begin{aligned}
& \rho A_{c} \frac{\partial^{2} u}{\partial t^{2}}=\frac{\partial}{\partial x}\left[A_{c} \tilde{g}_{e}\left(\frac{\partial u}{\partial x}\right)+A_{c} Y(0) g_{v d}\left(\frac{\partial u}{\partial x}\right)+A_{c} \int_{t-r}^{t} \dot{Y}(t-s) g_{v}\left(\frac{\partial u}{\partial x}\right) d s\right. \\
& \left.+A_{c} \sum_{k=1}^{K} Y\left(t-t_{k}\right)(-1)^{k+1}\left[g_{v i}\left(\frac{\partial u}{\partial x}\left(t_{k}\right)\right)-g_{v d}\left(\frac{\partial u}{\partial x}\left(t_{k}\right)\right)\right]\right] \quad \text { for } 0<x<\ell \\
& A_{c}\left[\tilde{g}_{e}\left(\frac{\partial u}{\partial x}(t)\right)+Y(0) g_{v d}\left(\frac{\partial u}{\partial x}(t)\right)+\int_{t-r}^{t} \dot{Y}(t-s) g_{v}\left(\frac{\partial u}{\partial x}(s)\right) d s\right. \\
& \left.+\sum_{k=1}^{K} Y\left(t-t_{k}\right)(-1)^{k+1}\left[g_{v i}\left(\frac{\partial u}{\partial x}\left(t_{k}\right)\right)-g_{v d}\left(\frac{\partial u}{\partial x}\left(t_{k}\right)\right)\right]\right]\left.\right|_{x=\ell}=f(t) \\
& u(t, 0)=0 \\
& u(0, x)=\varphi_{0} \\
& u_{t}(0, x)=0 \\
& u(t, x)=\varphi_{1}, \quad t<0 .
\end{aligned}
$$

We assume that the rod begins its motion at rest with possible deformation $\varphi_{0}$, fixed end at $x=0$, and memory $\varphi_{1}$. We also suppose that $\tilde{g}_{e}(\xi)=\xi+g_{e}(\xi)$, and include an (internal) Kelvin-Voigt damping term $\varepsilon u_{x x t}(\varepsilon>0)$ in the model. Then the system in variational form is:

$$
\begin{aligned}
& \rho A_{c} \ddot{u}-\varepsilon A_{c} \dot{u}_{x x}-A_{c} \frac{\partial}{\partial x}\left(\frac{\partial u}{\partial x}+g_{e}\left(\frac{\partial u}{\partial x}\right)+Y(0) g_{v d}\left(\frac{\partial u}{\partial x}\right)+\int_{t-r}^{t} \dot{Y}(t-s) g_{v}\left(\frac{\partial u}{\partial x}(s)\right) d s\right. \\
& \left.+\sum_{k=1}^{K} Y\left(t-t_{k}\right)(-1)^{k+1}\left[g_{v i}\left(\frac{\partial u}{\partial x}\left(t_{k}\right)\right)-g_{v d}\left(\frac{\partial u}{\partial x}\left(t_{k}\right)\right)\right]\right)=F(t) \\
& u(t, 0)=0 \\
& u(0, x)=\varphi_{0} \\
& u_{t}(0, x)=0 \\
& u(t, x)=\varphi_{1}, \quad t<0
\end{aligned}
$$


where (1.21) holds in the sense of $V^{*}$, where $V$ is an appropriately chosen Hilbert space.

In the following sections we shall show that, under certain assumptions, the system (1.21)(1.25) has a unique global weak solution. The study of Boltzmann type constitutive laws is, of course, not new and similar stress-strain laws, e.g.,

$$
\sigma(t, x)=\phi(\epsilon(t, x))+\int_{-\infty}^{t} a(t-s) \psi(\epsilon(s, x)) d s
$$

along with the resulting equations were studied by numerous other authors. To summarize a few related results, we note that it is known that in case the kernel $a$ is regular, globally defined smooth solutions exist for sufficiently smooth and small data. However, smooth solutions can develop singularities in finite time if the data are suitably large $[15,17,18,27$, 35]. The existence of weak solutions was shown in a special case in [29]. It appears that if the kernel $a$ is singular at zero, more regular existence properties can be established. (The use of singular kernels is supported by several investigators in polymer physics, e.g., Doi and Edwards [16], Rouse [32], Zimm [36], and Laun [23].) In this case, existence of global weak solutions was obtained in [2, 12, 19, 20, 28], while in [21] under some suitable assumptions the author shows that global smooth solutions exist for arbitrary data. A good survey of results concerning viscoelastic models can be found in the monograph [34].

\section{Existence of weak solutions}

We are going to consider the system

$$
\begin{aligned}
& u_{t t}-\varepsilon u_{x x t}-\frac{\partial}{\partial x}\left(\frac{\partial u}{\partial x}+g_{e}\left(\frac{\partial u}{\partial x}\right)+Y(0) g_{v}\left(\frac{\partial u}{\partial x}\right)+\int_{t-r}^{t} \dot{Y}(t-s) g_{v}\left(\frac{\partial u}{\partial x}(s)\right) d s\right. \\
& \left.+\sum_{k=1}^{K} Y\left(t-t_{k}\right)(-1)^{k+1}\left[g_{v i}\left(\frac{\partial u}{\partial x}\left(t_{k}\right)\right)-g_{v d}\left(\frac{\partial u}{\partial x}\left(t_{k}\right)\right)\right]\right)=F(t) \quad \text { in } V^{*} \\
& u(t, 0)=0 \\
& u(a, x)=\varphi_{0} \\
& u_{t}(a, x)=0 \\
& u(t, x)=\varphi_{1}, \quad t<a,
\end{aligned}
$$

for $a \leq t_{K} \leq t \leq t_{K+1}=b$. Our strategy in proving the existence of weak solutions is to consider the intervals $\left[t_{0}, t_{1}\right],\left[t_{1}, t_{2}\right], \ldots,\left[t_{K}, t_{K+1}\right]$ consecutively $\left(t_{0}=0\right)$. On $\left[t_{0}, t_{1}\right]$ the rubber is loading, $g_{v}=g_{v i}$ and there are no jump terms present. First we are going to show that a unique weak solution $u^{(1)}$ exists on this interval and is smooth enough so that 
$u^{(1)}\left(t_{1}\right), \quad \frac{\partial u^{(1)}}{\partial x}\left(t_{1}\right)$ exist. Next we suppose that a unique weak solution $u^{(K)}(t)$ exists on $\left[0, t_{K}\right]$ and we consider the interval $\left[t_{K}, t_{K+1}\right]$. There we have a similar system as above except that now we pick up a jump term and $\varphi_{0}$ and $\varphi_{1}$ are modified, i.e., $\tilde{\varphi}_{0}=u^{(K)}\left(t_{K}\right)$ and

$$
\tilde{\varphi}_{1}=\left\{\begin{array}{ll}
\varphi_{1} & \text { if } t<0 \\
u^{(K)} & \text { if } 0<t<t_{K} .
\end{array}\right\}
$$

We will again show that a unique weak solution $\tilde{u}$ exists on this interval with the necessary smoothness and

$$
u^{(K+1)}(t)=\left\{\begin{array}{ll}
u^{(K)} & \text { if } t \leq t_{K} \\
\tilde{u} & \text { if } t_{K}<t<t_{K+1} .
\end{array}\right\}
$$

is a weak solution on $\left[0, t_{K+1}\right]$.

We choose $V=H_{L}^{1}(0, \ell)=\left\{\phi: \phi \in H^{1}(0, \ell), \phi(0)=0\right\}$ and $H=L^{2}(0, \ell)$, so we have the Gelfand triple $V \hookrightarrow H \hookrightarrow V^{*} \cdot\langle\cdot, \cdot\rangle$ denotes the inner product in $H$, while $\langle\cdot, \cdot\rangle_{V^{*}, V}$ stands for the usual duality product. Also, there exist $k, \tilde{k}$ such that

$$
\tilde{k}\left\|\phi_{x}\right\|^{2} \leq\|\phi\|_{V}^{2} \leq k\left\|\phi_{x}\right\|^{2}
$$

for every $\phi \in V$. Let $\mathcal{L}_{[a, b]}$ denote the space of functions $u:[a, b] \rightarrow H$ such that

$$
\mathcal{L}_{[a, b]}=\left\{u: u \in L^{2}(a, b ; V), \quad u_{t} \in L^{2}(a, b ; V)\right\}
$$

We say that $u \in \mathcal{L}_{[a, b]}$ is a weak solution of (2.1)-(2.5) with $\varphi_{0} \in V$ and $\varphi_{1} \in L^{2}(a-r, a ; V)$ if it satisfies

$$
\begin{aligned}
& \int_{a}^{t}\left[-\left\langle u_{t}, \varphi_{t}\right\rangle+\left\langle u_{x}, \varphi_{x}\right\rangle+\varepsilon\left\langle u_{x t}, \varphi_{x}\right\rangle+\left\langle g_{e}\left(u_{x}\right), \varphi_{x}\right\rangle+\left\langle Y(0) g_{v}\left(u_{x}, \dot{u}_{x}\right), \varphi_{x}\right\rangle\right. \\
& +\left\langle\sum_{k=1}^{K} H\left(\tau-t_{k}\right) Y\left(\tau-t_{k}\right)(-1)^{k+1}\left[g_{v i}\left(u_{x}\left(t_{k}\right)\right)-g_{v d}\left(u_{x}\left(t_{k}\right)\right)\right], \varphi_{x}\right\rangle d \tau \\
& \left.+\left\langle\int_{\tau-r}^{\tau} \dot{Y}(\tau-s) g_{v}\left(u_{x}, \dot{u}_{x}\right) d s, \varphi_{x}\right\rangle\right]+\left\langle u_{t}(t), \varphi\right\rangle=\int_{a}^{t}\langle F, \varphi\rangle_{V^{*}, V} d \tau
\end{aligned}
$$

for any $a \leq t_{K} \leq t \leq t_{K+1}=b$ and $\varphi \in \mathcal{L}_{[a, b]}$ as well as the initial condition

$$
u(a, x)=\varphi_{0}
$$

Here

$$
H(s)=\left\{\begin{array}{ll}
1 & \text { if } s \geq 0 \\
0 & \text { if } s<0
\end{array}\right\}
$$




\subsection{Assumptions and Main Result}

We make the following assumptions (these assumptions on the nonlinear term are the same as in [6], except that we require that a strict monotonicity condition hold for $g_{e}+g_{v}$.):

A1) $g_{e}, g_{v i}$ and $g_{v d}$ are continuous nonlinear mappings of real gradient type, i.e., $g_{e}, g_{v i}, g_{v d}$ : $H \rightarrow H$ such that there exist continuous Frechet differentiable nonlinear functionals $G_{e}, G_{v i}, G_{v d}: H \rightarrow \mathbb{R}$ with

$$
\begin{aligned}
& G_{e}^{\prime}(\varphi) \psi=\operatorname{Re}\left\langle g_{e}(\varphi), \psi\right\rangle \\
& G_{v i}^{\prime}(\varphi) \psi=\operatorname{Re}\left\langle g_{v i}(\varphi), \psi\right\rangle \\
& G_{v d}^{\prime}(\varphi) \psi=\operatorname{Re}\left\langle g_{v d}(\varphi), \psi\right\rangle
\end{aligned}
$$

for every $\psi \in H$.

A2) There exist constants $C_{1}^{e}, C_{1}^{v i}, C_{1}^{v d}, C_{2}^{e}, C_{2}^{v i}, C_{2}^{v d}, C_{3}^{e}, C_{3}^{v i}, C_{3}^{v d}, \nu^{e}, \nu^{v i}, \nu^{v d}>0$ such that

$$
\begin{aligned}
& -\frac{\tilde{k}}{4}\left(k^{-1}-\nu^{e}\right)\|\varphi\|^{2}-C_{1}^{e} \leq G_{e}(\varphi) \leq C_{2}^{e}\|\varphi\|^{2}+C_{3}^{e} \\
& -\frac{\tilde{k}}{4}\left(k^{-1}-\nu^{v i}\right)\|\varphi\|^{2}-C_{1}^{v i} \leq G_{v i}(\varphi) \leq C_{2}^{v i}\|\varphi\|^{2}+C_{3}^{v i} \\
& -\frac{\tilde{k}}{4}\left(k^{-1}-\nu^{v d}\right)\|\varphi\|^{2}-C_{1}^{v d} \leq G_{v d}(\varphi) \leq C_{2}^{v d}\|\varphi\|^{2}+C_{3}^{v d}
\end{aligned}
$$

A3) The nonlinear functions $g_{e}, g_{v i}, g_{v d}$ satisfy

$$
\begin{aligned}
& \left\|g_{e}(\varphi)\right\| \leq \tilde{C}_{1}^{e}\|\varphi\|+\tilde{C}_{2}^{e} \\
& \left\|g_{v i}(\varphi)\right\| \leq \tilde{C}_{1}^{v i}\|\varphi\|+\tilde{C}_{2}^{v i} \\
& \left\|g_{v d}(\varphi)\right\| \leq \tilde{C}_{1}^{v d}\|\varphi\|+\tilde{C}_{2}^{v d}
\end{aligned}
$$

for every $\varphi \in H$, for some constants $\tilde{C}_{1}^{e}, \tilde{C}_{2}^{e}, \tilde{C}_{1}^{v i}, \tilde{C}_{2}^{v i}, \tilde{C}_{1}^{v d}, \tilde{C}_{2}^{v d}$.

A4) The Frechet derivatives of $g_{e}, g_{v i}$ and $g_{v d}$ exist and satisfy

$$
\begin{aligned}
& g_{e}^{\prime}(\varphi) \in \mathcal{L}(H, H) \text { with }\left\|g_{e}^{\prime}(\varphi)\right\|_{\mathcal{L}(H, H)} \leq \tilde{C}_{3}^{e}, \\
& g_{v i}^{\prime}(\varphi) \in \mathcal{L}(H, H) \text { with }\left\|g_{v i}^{\prime}(\varphi)\right\|_{\mathcal{L}(H, H)} \leq \tilde{C}_{3}^{v i} \text { and } \\
& g_{v d}^{\prime}(\varphi) \in \mathcal{L}(H, H) \text { with }\left\|g_{v d}^{\prime}(\varphi)\right\|_{\mathcal{L}(H, H)} \leq \tilde{C}_{3}^{v d} .
\end{aligned}
$$


A5) We suppose that the following monotonicity conditions hold for every $\varphi, \psi \in H$, with $\mu>0$ :

$$
\begin{aligned}
& \operatorname{Re}\left\langle g_{e}(\varphi)-g_{e}(\psi), \varphi-\psi\right\rangle+\operatorname{Re}\left\langle g_{v i}(\varphi)-g_{v i}(\psi), \varphi-\psi\right\rangle \geq \mu\|\varphi-\psi\|^{2} \\
& \operatorname{Re}\left\langle g_{e}(\varphi)-g_{e}(\psi), \varphi-\psi\right\rangle+\operatorname{Re}\left\langle g_{v d}(\varphi)-g_{v d}(\psi), \varphi-\psi\right\rangle \geq \mu\|\varphi-\psi\|^{2}
\end{aligned}
$$

A6) We assume that $Y$ is a smooth memory kernel such that there exist constants $C_{4}, C_{5}, C_{6}$ such that

$$
|Y(t)| \leq C_{4}, \quad|\dot{Y}(t)| \leq C_{5}, \quad|\ddot{Y}(t)| \leq C_{6}
$$

for $-r \leq t \leq t_{\max }$.

A7) The forcing term $F \in L^{2}\left(0, t_{\max } ; V^{*}\right)$.

Our main result is the following:

Theorem 2.1 Under assumptions A1)-A7) there exists a unique global weak solution of (2.1)-(2.5) for any $\varphi_{0} \in V, \varphi_{1} \in L^{2}(a-r, a ; V)$.

We first prove that under these assumptions a weak solution $u \in \mathcal{L}_{\left[0, t_{1}\right]}$ exists for any $\varphi_{0} \in V$ and $\varphi_{1} \in L^{2}(-r, 0 ; V)$, i.e., we use $a=t_{0}=0, b=t_{1}$ in our definition of the weak solution. The method of the proof is very similar to the one in [6]. First we give an apriori estimate, then introduce Galerkin approximations to the weak solution and justify taking the limit. The monotonicity condition (2.21) plays a major role in showing that the limit of the Galerkin approximates is a weak solution. Next we show that this solution can be continued on $\left[t_{1}, t_{2}\right]$, etc.

\section{The weak solution on $\left[0, t_{1}\right]$}

In this section we consider the interval $\left[0, t_{1}\right]$, where the rubber is loading for the first time, $g_{v}=g_{v i}$ and no jump terms are present. Thus we have the following system:

$$
\begin{aligned}
& u_{t t}-\varepsilon u_{x x t}-\frac{\partial}{\partial x}\left(\frac{\partial u}{\partial x}+g_{e}\left(\frac{\partial u}{\partial x}\right)+Y(0) g_{v i}\left(\frac{\partial u}{\partial x}\right)+\int_{t-r}^{t} \dot{Y}(t-s) g_{v}\left(\frac{\partial u}{\partial x}(s)\right) d s\right) \\
& =F(t) \quad \text { in } V^{*} \\
& u(t, 0)=0
\end{aligned}
$$




$$
\begin{aligned}
& u(0, x)=\varphi_{0} \\
& u_{t}(0, x)=0 \\
& u(t, x)=\varphi_{1}, \quad t<0,
\end{aligned}
$$

\subsection{The apriori estimate}

Let us assume that $Y(0)=1$. We choose $\varphi=u_{t}$ and take the inner product of (3.24) with $\varphi$.

$$
\begin{aligned}
& \left\langle u_{t t}, u_{t}\right\rangle_{V^{*}, V}+\left\langle u_{x}, u_{t x}\right\rangle+\varepsilon\left\langle u_{t x}, u_{t x}\right\rangle+\left\langle g_{e}\left(u_{x}\right), u_{t x}\right\rangle+\left\langle g_{v i}\left(u_{x}\right), u_{t x}\right\rangle \\
& +\left\langle\int_{t-r}^{t} \dot{Y}(t-s) g_{v}\left(u_{x}\right) d s, u_{t x}\right\rangle=\left\langle F(t), u_{t}\right\rangle_{V^{*}, V}
\end{aligned}
$$

Taking the real part and using $(2.9),(2.10)$ we get:

$$
\begin{aligned}
& \frac{d}{d t}\left[\frac{1}{2}\left\|u_{t}(t)\right\|^{2}+\frac{1}{2}\left\|u_{x}\right\|^{2}+G_{e}\left(u_{x}\right)+G_{v i}\left(u_{x}\right)\right]+\varepsilon\left\langle u_{t x}, u_{t x}\right\rangle \\
& +\operatorname{Re}\left\langle\int_{t-r}^{t} \dot{Y}(t-s) g_{v}\left(u_{x}\right) d s, u_{t x}\right\rangle=\operatorname{Re}\left\langle F(t), u_{t}\right\rangle_{V^{*}, V}
\end{aligned}
$$

Integrating from 0 to $t$, (where $t \leq t_{1}$ ), and using (2.6), (2.12),(2.13) we obtain

$$
\begin{aligned}
& \left\|u_{t}(t)\right\|^{2}+\nu\|u\|_{V}^{2}+2 \varepsilon \int_{0}^{t}\left\|u_{t x}\right\|^{2} d \tau+2 \int_{0}^{t} \operatorname{Re}\left\langle\int_{0}^{\tau} \dot{Y}(\tau-s) g_{v i}\left(u_{x}\right) d s, u_{x t}\right\rangle d \tau \leq M_{2} \\
& +M_{1}\left\|\varphi_{0}\right\|_{V}^{2}+2 \int_{0}^{t} \operatorname{Re}\left\langle F, u_{t}\right\rangle_{V^{*}, V} d \tau-2 \int_{0}^{t} \operatorname{Re}\left\langle\int_{\tau-r}^{0} \dot{Y}(\tau-s) g_{v i}\left(\varphi_{1 x}\right) d s, u_{t x}\right\rangle d \tau,
\end{aligned}
$$

where $\nu=\frac{\tilde{k}}{2 k}\left(\nu^{e}+\nu^{v i}\right)$. Note that we have separated the hysteresis integral into two terms assuming without loss of generality that $r>t$. Thus,

$$
\left\|u_{t}(t)\right\|^{2}+\nu\|u\|_{V}^{2}+\tilde{\varepsilon} \int_{0}^{t}\left\|u_{t}\right\|_{V}^{2} d \tau \leq M_{2}+M_{1}\left\|\varphi_{0}\right\|^{2}-2 I_{1}+2 I_{2}-2 I_{3},
$$

where

$$
\begin{aligned}
& I_{1}=\int_{0}^{t} \operatorname{Re}\left\langle\int_{0}^{\tau} \dot{Y}(\tau-s) g_{v i}\left(u_{x}\right) d s, u_{x t}\right\rangle d \tau, \\
& I_{2}=\int_{0}^{t} \operatorname{Re}\left\langle F, u_{t}\right\rangle_{V^{*}, V} d \tau \text { and } \\
& I_{3}=\int_{0}^{t} \operatorname{Re}\left\langle\int_{\tau-r}^{0} \dot{Y}(\tau-s) g_{v}\left(\varphi_{1 x}\right) d s, u_{t x}\right\rangle d \tau .
\end{aligned}
$$


First we estimate $I_{1}$. Let $M(\tau)=\int_{0}^{\tau} \dot{Y}(\tau-s) g_{v i}\left(u_{x}\right) d s$.

$$
\begin{aligned}
& \left|I_{1}\right|=\left|\int_{0}^{t} \operatorname{Re}\left\langle M(\tau), \frac{d}{d \tau} u_{x}\right\rangle d \tau\right| \leq\left|\left\langle M(t), u_{x}\right\rangle-\int_{0}^{t}\left\langle\frac{d}{d \tau} M(\tau), u_{x}\right\rangle d \tau\right| \leq \frac{1}{4 \delta}\|M(t)\|^{2} \\
& +\delta\left\|u_{x}\right\|^{2}+\left|\int_{0}^{t}\left(\left\langle\dot{Y}(0) g_{v i}\left(u_{x}(\tau)\right), u_{x}(\tau)\right\rangle+\left\langle\int_{0}^{\tau} \ddot{Y}(\tau-s) g_{v i}\left(u_{x}(s)\right) d s, u_{x}(\tau)\right\rangle\right) d \tau\right|
\end{aligned}
$$

Estimating the integral, we have

$$
\begin{aligned}
& \left|\int_{0}^{t}\left\langle\dot{Y}(0) g_{v i}\left(u_{x}(\tau)\right), u_{x}(\tau)\right\rangle d \tau\right| \leq|\dot{Y}(0)| \int_{0}^{t}\left\|g_{v i}\left(u_{x}\right)\right\|\left\|u_{x}\right\| d \tau \\
& \leq|\dot{Y}(0)| \int_{0}^{t}\left(\tilde{C}_{1}^{v i}\left\|u_{x}\right\|+\tilde{C}_{2}^{v i}\right)\left\|u_{x}\right\| d \tau \leq M_{3} \int_{0}^{t}\|u\|_{V}^{2} d \tau+M_{4}
\end{aligned}
$$

and

$$
\begin{aligned}
& \left|\int_{0}^{t}\left\langle\int_{0}^{\tau} \ddot{Y}(\tau-s) g_{v i}\left(u_{x}(s)\right) d s, u_{x}(\tau)\right\rangle d \tau\right| \leq \int_{0}^{t}\left\|\int_{0}^{\tau} \ddot{Y}(\tau-s) g_{v i}\left(u_{x}\right) d s\right\|\left\|u_{x}(\tau)\right\| d \tau \\
& \leq \int_{0}^{t} \int_{0}^{\tau} C_{6}\left(\tilde{C}_{1}^{v i}\left\|u_{x}\right\|+\tilde{C}_{2}^{v i}\right) d s\left\|u_{x}\right\| d \tau \leq C_{6} \tilde{C}_{1}^{v i}\left(\int_{0}^{t}\left\|u_{x}\right\| d \tau\right)^{2}+C_{7} \tilde{C}_{2}^{v i} \int_{0}^{t}\left\|u_{x}\right\| d \tau \\
& \leq M_{5} \int_{0}^{t}\|u\|_{V}^{2} d \tau+M_{6} .
\end{aligned}
$$

We use a standard estimate for $I_{2}$ :

$$
\left|I_{2}\right| \leq \gamma \int_{0}^{t}\left\|u_{t}\right\|_{V}^{2} d \tau+\frac{1}{4 \gamma} \int_{0}^{t}\|F\|_{V^{*}}^{2} d \tau
$$

where $\gamma>0$ is chosen such that $2 \gamma<\tilde{\varepsilon}$. Let $\tilde{M}(\tau)=\int_{\tau-r}^{0} \dot{Y}(\tau-s) g_{v}\left(\left(\varphi_{1}\right)_{x}\right) d s$. We have

$$
\begin{aligned}
& \left|I_{3}\right|=\left|\int_{0}^{t} \operatorname{Re}\left\langle\tilde{M}(\tau), u_{t x}\right\rangle d \tau\right| \leq\left|\left\langle\tilde{M}(\tau), u_{x}\right\rangle\right|_{\tau=0}^{\tau=t}-\int_{0}^{t}\left\langle\frac{\partial}{\partial t} \tilde{M}(\tau), u_{x}\right\rangle d \tau \mid \\
& \leq \frac{1}{4 \alpha}\|\tilde{M}(t)\|^{2}+\alpha\left\|u_{x}\right\|^{2}+\frac{1}{4 \beta}\|\tilde{M}(0)\|^{2}+\beta\left\|\left(\varphi_{0}\right)_{x}\right\|^{2} \\
& +\int_{0}^{t}\left|\left\langle\int_{\tau-r}^{0} \ddot{Y}(\tau-s) g_{v}\left(\left(\varphi_{1}\right)_{x}\right) d s, u_{x}\right\rangle\right| d \tau \leq \int_{0}^{t}\left\|\int_{\tau-r}^{0} \ddot{Y}(\tau-s) g_{v}\left(\left(\varphi_{1}\right)_{x}\right) d s\right\|\left\|u_{x}(\tau)\right\| d \tau \\
& +\frac{1}{4 \alpha}\|\tilde{M}(t)\|^{2}+\alpha\left\|u_{x}\right\|^{2}+\frac{1}{4 \beta}\|\tilde{M}(0)\|^{2}+\beta\left\|\left(\varphi_{0}\right)_{x}\right\|^{2} \leq M_{7} \int_{0}^{t}\|u\|_{V}^{2} d \tau \\
& +M_{8}+\frac{1}{4 \alpha}\|\tilde{M}(t)\|^{2}+\alpha \tilde{k}^{-1}\|u\|_{V}^{2}+\frac{1}{4 \beta}\|\tilde{M}(0)\|^{2} \\
& +\beta \tilde{k}^{-1}\left\|\varphi_{0}\right\|_{V}^{2} .
\end{aligned}
$$


Since

$$
\begin{aligned}
& \|M(t)\|^{2}=\left\|\int_{0}^{t} \dot{Y}(t-s) g_{v i}\left(u_{x}\right) d s\right\|^{2} \leq\left(C_{5} \int_{0}^{t} \tilde{C}_{1}^{v i}\left\|u_{x}\right\|+\tilde{C}_{2}^{v i} d \tau\right)^{2} \\
& \leq M_{9} \int_{0}^{t}\|u\|_{V}^{2} d \tau+M_{10} \text { and } \\
& \|\tilde{M}(t)\| \leq M_{11}
\end{aligned}
$$

by (3.32)-(3.36), we have that (3.31) yields

$$
\left\|u_{t}(t)\right\|^{2}+\tilde{\nu}\|u\|_{V}^{2}+\theta \int_{0}^{t}\left\|u_{t}\right\|_{V}^{2} d \tau \leq C\left(\varphi_{0}, \varphi_{1}, F, t_{1}\right)+M_{12} \int_{0}^{t}\|u(\tau)\|_{V}^{2} d \tau
$$

for $\alpha, \delta, \gamma$ small enough, $(\theta=\tilde{\varepsilon}-2 \gamma)$. By Gronwall's inequality, it follows that

$$
\left\|u_{t}(t)\right\|^{2}+\tilde{\nu}\|u(t)\|_{V}^{2}+\theta \int_{0}^{t}\left\|u_{t}\right\|_{V}^{2} d \tau \leq \tilde{C}\left(\varphi_{0}, \varphi_{1}, F, t_{1}\right) .
$$

\subsection{Galerkin approximations and their convergence}

Let $\left\{\psi_{k}\right\}_{k=1}^{\infty} \subset V$ be an orthonormal system in $V$. We define Galerkin approximations for (3.24) by

$$
u^{N}(t)=\sum_{k=1}^{N} c_{k}^{N}(t) \psi_{k},
$$

where $\left\{c_{k}^{N}(t)\right\}$ are chosen so that $u^{N}(t)$ satisfies

$$
\begin{aligned}
& \frac{d^{2}}{d t^{2}}\left\langle u^{N}(t), \psi_{j}\right\rangle+\left\langle u_{x}^{N}, \psi_{j x}\right\rangle+\varepsilon \frac{d}{d t}\left\langle u_{x}^{N}, \psi_{j x}\right\rangle+\left\langle g_{e}\left(u_{x}^{N}\right), \psi_{j x}\right\rangle \\
& +\left\langle g_{v i}\left(u_{x}^{N}\right), \psi_{j x}\right\rangle+\left\langle\int_{t-r}^{t} \dot{Y}(t-s) g_{v}\left(u_{x}^{N}\right) d s, \psi_{j x}\right\rangle=\left\langle F, \psi_{j}\right\rangle_{V^{*}, V} \\
& \text { for } j=1, \ldots, N, \quad \text { with initial conditions } \\
& c_{k}^{N}(0)=c_{0 k}^{N}, \quad \frac{d}{d t} c_{k}^{N}(0)=0 .
\end{aligned}
$$

We choose $\left\{c_{0 k}^{N}\right\}$ so that $\lim _{N \rightarrow \infty} \sum_{1}^{\infty} c_{0 k}^{N} \psi_{k}=\varphi_{0}$ in $V$. Arguing in the usual way we obtain that the Galerkin approximations also satisfy (3.39), i.e.,

$$
\left\|u_{t}^{N}(t)\right\|^{2}+\tilde{\nu}\left\|u^{N}(t)\right\|_{V}^{2}+\theta \int_{0}^{t}\left\|u_{t}^{N}\right\|_{V}^{2} d \tau \leq \tilde{C}
$$

where $\tilde{C}$ is independent of $N$. 
It follows from (3.42) that $\left\{u^{N}\right\}$ is bounded in $C\left(0, t_{1} ; V\right) \subset L^{2}\left(0, t_{1} ; V\right)$, and $\left\{u_{t}^{N}\right\}$ is bounded in $C\left(0, t_{1} ; H\right)$ and in $L^{2}\left(0, t_{1} ; V\right)$. Thus, there exists a subsequence (denoted again by $u^{N}$ ) such that

$$
\begin{aligned}
& u^{N} \rightarrow u \text { weakly in } L^{2}\left(0, t_{1} ; V\right) \\
& u_{t}^{N} \rightarrow u_{t} \text { weakly in } L^{2}\left(0, t_{1} ; V\right) .
\end{aligned}
$$

As in [6] we can show that there exist a subsequence $u^{N}$ and $u \in \mathcal{L}_{\left[0, t_{1}\right]}$ such that

a) The set $\left\{u^{N}\right\}$ is equicontinuous and bounded in $C\left(0, t_{1} ; V\right)$ and

$$
u^{N}(t) \rightarrow u(t) \text { weakly in } V
$$

uniformly in $t \in\left[0, t_{1}\right]$, i.e., $u^{N} \rightarrow u$ in $C_{W}\left(0, t_{1} ; V\right)$;

b) The set $\left\{u_{t}^{N}\right\}$ is bounded in $C\left(0, t_{1} ; H\right)$, equicontinuous in $C_{W}\left(0, t_{1} ; H\right)$ and

$$
u_{t}^{N}(t) \rightarrow u_{t}(t) \text { weakly in } H
$$

uniformly in $t \in\left[0, t_{1}\right]$;

c)

$$
u_{t}^{N} \rightarrow u_{t} \text { strongly in } L^{2}\left(0, t_{1} ; H\right)
$$

d) There exist $h_{e}, h_{v i} \in L^{2}\left(0, t_{1} ; H\right)$ such that

$$
\begin{aligned}
& g_{e}\left(u_{x}^{N}\right) \rightarrow h_{e} \text { weakly in } L^{2}\left(0, t_{1} ; H\right), \\
& g_{v i}\left(u_{x}^{N}\right) \rightarrow h_{v i} \text { weakly in } L^{2}\left(0, t_{1} ; H\right) .
\end{aligned}
$$

(3.48) and We note that (3.49) follow from (3.42), (2.6) and (2.15), (2.16). We can prove (3.45) exactly as in [6] using a general version of the Ascoli-Arzela Theorem [30]. The same technique can be used to show the statement (3.46), once equicontinuity of $\left\{u_{t}^{N}\right\}$ is established. The key to this (as in [6]) is the boundedness of the set $\left\{u_{t t}^{N}\right\}$ in $L^{2}\left(0, t_{1} ; V^{*}\right)$ : For a fixed $M$, let $\Phi_{M}=\sum_{k=1}^{M} a_{k}(t) \psi_{k}, a_{k} \in C^{1}\left[0, t_{1}\right]$. For $N \geq M$ we have

$$
\begin{aligned}
& \left|u_{t t}^{N}\left(\Phi_{M}\right)\right|=\left|\int_{0}^{t_{1}}\left\langle u_{t t}^{N}(\tau), \Phi_{M}(\tau)\right\rangle_{V^{*}, V} d \tau\right| \leq \mid \int_{0}^{t_{1}}\left[-\left\langle u_{x}^{N}, \Phi_{M x}\right\rangle-\varepsilon\left\langle u_{t x}^{N}, \Phi_{M x}\right\rangle\right. \\
& -\left\langle g_{e}\left(u_{x}^{N}\right), \Phi_{M x}\right\rangle-\left\langle g_{v i}\left(u_{x}^{N}\right), \Phi_{M x}\right\rangle-\left\langle\int_{0}^{\tau} \dot{Y}(\tau-s) g_{v i}\left(u_{x}^{N}\right) d s, \Phi_{M x}\right\rangle+\left\langle F, \Phi_{M}\right\rangle
\end{aligned}
$$




$$
\begin{aligned}
& \left.-\left\langle\int_{\tau-r}^{0} \dot{Y}(\tau-s) g_{v}\left(\varphi_{1 x}\right) d s, \Phi_{M x}\right\rangle\right] d \tau \mid \leq m_{1} \int_{0}^{t_{1}}\left\|u^{N}(\tau)\right\|_{V}\left\|\Phi_{M}\right\|_{V} d \tau \\
& +m_{2} \int_{0}^{t_{1}}\left\|u_{t}^{N}(\tau)\right\|_{V}\left\|\Phi_{M}\right\|_{V} d \tau+m_{3} \int_{0}^{t_{1}}\left\|g_{e}\left(u_{x}^{N}\right)\right\|\left\|\Phi_{M}\right\|_{V} d \tau+m_{4} \int_{0}^{t_{1}}\left\|g_{v i}\left(u_{x}^{N}\right)\right\|\left\|\Phi_{M}\right\|_{V} d \tau \\
& +m_{5} \int_{0}^{t_{1}} \int_{0}^{t_{1}}\left\|g_{v i}\left(u_{x}^{N}\right)\right\| d s\left\|\Phi_{M}\right\|_{V} d \tau+\int_{0}^{t_{1}}\|F(\tau)\|_{V^{*}}\left\|\Phi_{M}\right\|_{V} d \tau+m_{6} \int_{0}^{t_{1}}\left\|\Phi_{M}\right\|_{V} d \tau \\
& \leq K\left\|\Phi_{M}\right\|_{L^{2}\left(0, t_{1} ; V\right)}
\end{aligned}
$$

By (3.42), $K$ does not depend on $N$ or $M$. ((3.50) is valid for $N<M$ also, by the orthogonality of $\left.\psi_{k}.\right)$ Equicontinuity can now be proved exactly as in [6].

Now since $\left\{u_{t}^{N}\right\}$ is bounded in $L^{2}\left(0, t_{1} ; V\right)$ and by (3.50) $\left\{u_{t t}^{N}\right\}$ is bounded in $L^{2}\left(0, t_{1} ; V^{*}\right)$, Aubin's lemma $([6,1,26])$ guarantees that the set $\left\{u_{t}^{N}\right\}$ is relatively compact in $L^{2}\left(0, t_{1} ; H\right)$. Therefore we can conclude that there exists a subsequence $u^{N}$ such that $u_{t}^{N} \rightarrow u_{t}$ strongly in $L^{2}\left(0, t_{1} ; H\right)$, which proves the statement (3.47). Thus the statements (3.43)-(3.49) are all established.

\subsection{The limit process}

Let $\mathcal{P}_{M}$ denote the class of functions $\eta \in \mathcal{L}_{\left[0, t_{1}\right]}$, which can be represented in the form

$$
\eta(t)=\sum_{k=1}^{M} a_{k}(t) \psi_{k},
$$

where $a_{k}(t) \in C^{1}\left[0, t_{1}\right]$. Then $\mathcal{P}=\cup_{M=1}^{\infty} \mathcal{P}_{M}$ is dense in $\mathcal{L}_{\left[0, t_{1}\right]}$. By definition the Galerkin approximations $u^{N}$ satisfy

$$
\begin{aligned}
& \int_{0}^{t}\left[-\left\langle u_{t}^{N}, \eta_{t}\right\rangle+\left\langle u_{x}^{N}, \eta_{x}\right\rangle+\varepsilon\left\langle u_{x t}^{N}, \eta_{x}\right\rangle+\left\langle g_{e}\left(u_{x}^{N}\right), \eta_{x}\right\rangle+\left\langle g_{v i}\left(u_{x}^{N}\right), \eta_{x}\right\rangle\right. \\
& \left.+\left\langle\int_{0}^{\tau} \dot{Y}(\tau-s) g_{v i}\left(u_{x}^{N}\right) d s, \eta_{x}\right\rangle\right] d \tau+\left\langle u_{t}^{N}(t), \eta\right\rangle=\int_{0}^{t}\langle F, \eta\rangle_{V^{*}, V} d \tau \\
& -\int_{0}^{t}\left\langle\int_{\tau-r}^{0} \dot{Y}(\tau-s) g_{v}\left(\varphi_{1 x}\right) d s, \eta_{x}\right\rangle d \tau
\end{aligned}
$$

for all $\eta \in \mathcal{P}_{M}$, for $M \leq N$. Let $\eta \in \mathcal{P}_{M}$ be fixed, with $M \leq N$, and take the limit in (3.51) as $N \rightarrow \infty$. We obtain

$$
\begin{aligned}
& \int_{0}^{t}\left[-\left\langle u_{t}, \eta_{t}\right\rangle+\left\langle u_{x}, \eta_{x}\right\rangle+\varepsilon\left\langle u_{x t}, \eta_{x}\right\rangle+\left\langle h_{e}, \eta_{x}\right\rangle+\left\langle h_{v i}, \eta_{x}\right\rangle\right. \\
& \left.+\left\langle\int_{0}^{\tau} \dot{Y}(\tau-s) h_{v i} d s, \eta_{x}\right\rangle\right] d \tau+\left\langle u_{t}(t), \eta\right\rangle=\int_{0}^{t}\langle F, \eta\rangle_{V^{*}, V} d \tau \\
& -\int_{0}^{t}\left\langle\int_{\tau-r}^{0} \dot{Y}(\tau-s) g_{v}\left(\varphi_{1 x}\right) d s, \eta_{x}\right\rangle d \tau
\end{aligned}
$$


We can argue that passage to the limit is possible in the hysteresis term since for given $t \in\left[0, t_{1}\right], \varphi \in H, \mathcal{N}: L^{2}(0, t ; H) \rightarrow \mathbb{R}$,

$$
\mathcal{N}(\psi)=\int_{0}^{t}\left\langle\int_{0}^{\tau} \dot{Y}(\tau-s) \psi(s) d s, \varphi\right\rangle d \tau
$$

is a bounded linear functional on $L^{2}(0, t ; H)$ and therefore weakly continuous. Consequently,

$$
\int_{0}^{t}\left\langle\int_{0}^{\tau} \dot{Y}(\tau-s) g_{v i}\left(u_{x}^{N}\right) d s, \eta_{x}\right\rangle d \tau \rightarrow \int_{0}^{t}\left\langle\int_{0}^{\tau} \dot{Y}(\tau-s) h_{v i}(s) d s, \eta_{x}\right\rangle d \tau
$$

by (3.49). Passage to the limit in all the other terms can be argued based on the convergences (3.43)-(3.49) (for details see [6]). Equation (3.52) holds for all $\eta \in \mathcal{P}_{M}$ for all $M$, and since $\mathcal{P}$ is dense in $\mathcal{L}_{\left[0, t_{1}\right]}$, for all $\eta \in \mathcal{L}_{\left[0, t_{1}\right]}$ as well.

To conclude that $u$ is indeed a weak solution, it remains to argue that for any $\eta \in \mathcal{L}_{\left[0, t_{1}\right]}$ and for $t \in\left[0, t_{1}\right]$

$$
\begin{aligned}
& \int_{0}^{t}\left[\left\langle g_{e}\left(u_{x}\right), \eta_{x}\right\rangle+\left\langle g_{v i}\left(u_{x}\right), \eta_{x}\right\rangle\right. \\
& \left.+\left\langle\int_{0}^{\tau} \dot{Y}(\tau-s) g_{v i}\left(u_{x}\right) d s, \eta_{x}\right\rangle\right] d \tau=\int_{0}^{t}\left[\left\langle h_{e}, \eta_{x}\right\rangle+\left\langle h_{v i}, \eta_{x}\right\rangle\right. \\
& \left.+\left\langle\int_{0}^{\tau} \dot{Y}(\tau-s) h_{v i} d s, \eta_{x}\right\rangle\right] d \tau
\end{aligned}
$$

First, we prove the following lemma.

Lemma 3.1 There exists $T_{0}$ with $t_{1} \geq T_{0}>0$ such that for any $t<T_{0}$ and any $\phi, \psi \in$ $L^{2}\left(0, t_{1} ; H\right)$ we have

$$
\begin{aligned}
& \int_{0}^{t} \operatorname{Re}\left[\left\langle g_{e}(\phi)-g_{e}(\psi), \phi-\psi\right\rangle+\left\langle g_{v i}(\phi)-g_{v i}(\psi), \phi-\psi\right\rangle\right. \\
& \left.+\left\langle\int_{0}^{\tau} \dot{Y}(\tau-s)\left(g_{v i}(\phi)-g_{v i}(\psi)\right) d s, \phi-\psi\right\rangle\right] d \tau \geq \frac{\mu}{2} \int_{0}^{t}\|\phi-\psi\|^{2} d \tau
\end{aligned}
$$

$T_{0}$ depends only on $\mu$ (from the monotonicity assumption (2.21)), on $\tilde{C}_{3}^{v i}$ from (2.19) and the bound $C_{5}$ on $\dot{Y}$.

Proof: By (2.21) we have

$$
\begin{aligned}
& \int_{0}^{t} \operatorname{Re}\left[\left\langle g_{e}(\phi)-g_{e}(\psi), \phi-\psi\right\rangle+\left\langle g_{v i}(\phi)-g_{v i}(\psi), \phi-\psi\right\rangle\right. \\
& \left.+\left\langle\int_{0}^{\tau} \dot{Y}(\tau-s)\left(g_{v i}(\phi)-g_{v i}(\psi)\right) d s, \phi-\psi\right\rangle\right] d \tau \geq \int_{0}^{t} \mu\|\phi-\psi\|^{2} d \tau \\
& +\int_{0}^{t}\left\langle\int_{0}^{\tau} \dot{Y}(\tau-s)\left(g_{v i}(\phi)-g_{v i}(\psi)\right) d s, \phi-\psi\right\rangle d \tau
\end{aligned}
$$


Now

$$
\begin{aligned}
& \left|\int_{0}^{t}\left\langle\int_{0}^{\tau} \dot{Y}(\tau-s)\left(g_{v i}(\phi(s))-g_{v i}(\psi(s))\right) d s, \phi(\tau)-\psi(\tau)\right\rangle d \tau\right|= \\
& \left|\int_{0}^{t}\left\langle\int_{0}^{\tau} \dot{Y}(\tau-s) \int_{0}^{1} g_{v i}^{\prime}(\theta \phi+(1-\theta) \psi)(\phi(s)-\psi(s)) d \theta d s, \phi(\tau)-\psi(\tau)\right\rangle d \tau\right| \\
& \leq \int_{0}^{t} \int_{0}^{\tau}|\dot{Y}(\tau-s)| \int_{0}^{1} \tilde{C}_{3}^{v i}\|\phi(s)-\psi(s)\| d \theta d s\|\phi(\tau)-\psi(\tau)\| d \tau \leq \tilde{C}_{3}^{v i} C_{5}\left(\int_{0}^{t}\|\phi-\psi\| d \tau\right)^{2} \\
& \leq \tilde{C}_{3}^{v i} C_{5} t \int_{0}^{t}\|\phi-\psi\|^{2} d \tau .
\end{aligned}
$$

Thus, for $\tilde{C}_{3}^{v i} C_{5} t \leq \frac{\mu}{2}$, i.e., for $t \leq \min \left(t_{1}, \frac{\mu}{2 \tilde{C}_{3}^{v i} C_{5}}\right)=T_{0}$, we have that (3.54) is satisfied. Thus Lemma 3.1 is proved.

Let $t \leq T_{0}, \eta=u^{N}$ in (3.51) and take the limit as $N \rightarrow \infty$. Using the convergences (3.43)-(3.49) and the weak lower semicontinuity of norms in a Hilbert space, the comparison with (3.52) yields

$$
\begin{aligned}
& \lim _{N \rightarrow \infty} \operatorname{Re} \int_{0}^{t}\left[\left\langle g_{e}\left(u_{x}^{N}\right), u_{x}^{N}\right\rangle\right. \\
& \left.+\left\langle g_{v i}\left(u_{x}^{N}\right), u_{x}^{N}\right\rangle+\left\langle\int_{0}^{\tau} \dot{Y}(\tau-s) g_{v i}\left(u_{x}^{N}\right) d s, u_{x}^{N}\right\rangle\right] d \tau \\
& \leq \int_{0}^{t}\left\langle h_{e}, u_{x}\right\rangle+\left\langle h_{v i}, u_{x}\right\rangle+\left\langle\int_{0}^{\tau} \dot{Y}(\tau-s) h_{v i} d s, u_{x}\right\rangle d \tau
\end{aligned}
$$

Now by Lemma 3.1 with $\phi=u_{x}^{N}$ and $\psi=\eta_{x}$ we have for $t \leq T_{0}$

$$
\begin{aligned}
& 0 \leq \frac{\mu}{2} \int_{0}^{t}\left\|u_{x}^{N}-\eta_{x}\right\|^{2} d \tau \\
& \leq \operatorname{Re} \int_{0}^{t}\left[\left\langle\left(g_{e}\left(u_{x}^{N}\right)-g_{e}\left(\eta_{x}\right)\right), u_{x}^{N}-\eta_{x}\right\rangle\right. \\
& +\left\langle\left(g_{v i}\left(u_{x}^{N}\right)-g_{v i}\left(\eta_{x}\right)\right), u_{x}^{N}-\eta_{x}\right\rangle \\
& \left.+\left\langle\int_{0}^{\tau} \dot{Y}(\tau-s) g_{v i}\left(u_{x}^{N}\right)-g_{v i}\left(\eta_{x}\right) d s, u_{x}^{N}-\eta_{x}\right\rangle\right] d \tau .
\end{aligned}
$$

Letting $N \rightarrow \infty$ in (3.58) and using the above relation (3.57) along with the standard Minty-Browder technique, we find

$$
\begin{aligned}
& \int_{0}^{t}\left[\left\langle g_{e}\left(u_{x}\right), \eta_{x}\right\rangle+\left\langle g_{v i}\left(u_{x}\right), \eta_{x}\right\rangle\right. \\
& \left.+\left\langle\int_{0}^{\tau} \dot{Y}(\tau-s) g_{v i}\left(u_{x}\right) d s, \eta_{x}\right\rangle\right] d \tau=\int_{0}^{t}\left[\left\langle h_{e}, \eta_{x}\right\rangle+\left\langle h_{v i}, \eta_{x}\right\rangle\right. \\
& \left.+\left\langle\int_{0}^{\tau} \dot{Y}(\tau-s) h_{v i} d s, \eta_{x}\right\rangle\right] d \tau
\end{aligned}
$$


for all $\eta \in \mathcal{L}_{\left[0, t_{1}\right]}$, which proves $(3.53)$ for $t \in\left[0, T_{0}\right]$. We note that it is possible to show that

$$
u_{x}^{N} \rightarrow u_{x} \quad \text { in } L^{2}\left(0, T_{0} ; H\right)
$$

by setting $\eta=u$ in relation (3.58) and passing to the limit as $N \rightarrow \infty$, again using (3.57). Note that this implies $u_{N} \rightarrow u$ strongly in $L^{2}\left(0, T_{0} ; V\right)$.

Thus, we verified the existence of a weak solution on $\left[0, T_{0}\right]$. Since $T_{0}$ depends only on constants from our assumptions (and independent of the solution itself) we can extend the weak solution to $\left[T_{0}, 2 T_{0}\right]$ etc. to obtain the weak solution $u^{(1)}$ on the interval $\left[0, t_{1}\right]$.

\subsection{Uniqueness of the weak solution on $\left[0, t_{1}\right]$}

Let $u$ and $\tilde{u}$ be weak solutions of $(3.24)$ on $\left[0, t_{1}\right]$ corresponding to the data $\varphi_{0}, \varphi_{1}, f$. Then $w=u-\tilde{u}$ satisfies $w(0)=w_{t}(0)=0, w(t, x)=0$ for $t<0$ and

$$
\begin{aligned}
& \left\langle w_{t t}, \eta\right\rangle_{V^{*}, V}+\left\langle w_{x}, \eta_{x}\right\rangle+\varepsilon\left\langle w_{t x}, \eta_{x}\right\rangle+\left\langle g_{e}\left(u_{x}\right), \eta_{x}\right\rangle \\
& +\left\langle g_{v i}\left(u_{x}\right), \eta_{x}\right\rangle+\left\langle\int_{0}^{t} \dot{Y}(t-s) g_{v i}\left(u_{x}\right) d s, \eta_{x}\right\rangle-\left\langle g_{e}\left(\tilde{u}_{x}\right), \eta_{x}\right\rangle-\left\langle g_{v i}\left(\tilde{u}_{x}\right), \eta_{x}\right\rangle \\
& -\left\langle\int_{0}^{t} \dot{Y}(t-s) g_{v i}\left(\tilde{u}_{x}\right) d s, \eta_{x}\right\rangle=0
\end{aligned}
$$

for all $\eta \in \mathcal{L}_{\left[0, t_{1}\right]}$ and for almost all $t \in\left[0, t_{1}\right]$, by the definition of the weak solution. Let

$$
\begin{aligned}
& \Delta g_{e}=g_{e}\left(u_{x}\right)-g_{e}\left(\tilde{u}_{x}\right) \\
& \Delta g_{v i}=g_{v i}\left(u_{x}\right)-g_{v i}\left(\tilde{u}_{x}\right) .
\end{aligned}
$$

Choosing $\eta=w_{t}$ in $(3.61)$ we formally $\left(w_{t} \notin \mathcal{L}_{\left[0, t_{1}\right]}\right)$ obtain:

$$
\begin{aligned}
& \frac{d}{d t}\left\{\frac{1}{2}\left\|w_{t}\right\|^{2}+\frac{1}{2}\left\|w_{x}\right\|^{2}\right\}+\varepsilon\left\|w_{t x}\right\|^{2}+\left\langle\Delta g_{e}, w_{t x}\right\rangle+\left\langle\Delta g_{v i}, w_{t x}\right\rangle \\
& +\left\langle\int_{0}^{\tau} \dot{Y}(\tau-s) \Delta g_{v i} d s, w_{t x}\right\rangle=0
\end{aligned}
$$

Integrating from 0 to $t,\left(t \leq t_{1}\right)$ we obtain:

$$
\begin{aligned}
& \left\|w_{t}(t)\right\|^{2}+k^{-1}\|w(t)\|_{V}^{2}+2 \varepsilon \int_{0}^{t}\left\|w_{t x}\right\|^{2} d \tau \\
& \leq-2 \int_{0}^{t}\left[\left\langle\Delta g_{e}, w_{t x}\right\rangle+\left\langle\Delta g_{v i}, w_{t x}\right\rangle+\left\langle\int_{0}^{\tau} \dot{Y}(\tau-s) \Delta g_{v} d s, w_{t x}\right\rangle\right] d \tau
\end{aligned}
$$


Now $\int_{0}^{t}\left\langle\Delta g_{e}, w_{t x}\right\rangle d \tau$ and $\int_{0}^{t}\left\langle\Delta g_{v}, w_{t x}\right\rangle d \tau$ can be estimated as in [6], i.e.,

$$
\left|\int_{0}^{t}\left\langle\Delta g_{e}, w_{t x}\right\rangle d \tau\right| \leq \tilde{C}_{3}^{e} \frac{1}{4 \delta} \tilde{k}^{-1} \int_{0}^{t}\|w(\tau)\|_{V}^{2} d \tau+\tilde{C}_{3}^{e} \delta \int_{0}^{t}\left\|w_{t x}\right\|^{2} d \tau
$$

and

$$
\left|\int_{0}^{t}\left\langle\Delta g_{v i}, w_{t x}\right\rangle d \tau\right| \leq \tilde{C}_{3}^{v i} \frac{1}{4 \delta} \tilde{k}^{-1} \int_{0}^{t}\|w(\tau)\|_{V}^{2} d \tau+\tilde{C}_{3}^{v i} \delta \int_{0}^{t}\left\|w_{t x}\right\|^{2} d \tau
$$

where $\delta>0$ is arbitrary. Also,

$$
\begin{aligned}
& \left|\int_{0}^{t}\left\langle\int_{0}^{\tau} \dot{Y}(\tau-s) \Delta g_{v i} d s, w_{t x}\right\rangle d \tau\right| \\
& =\left|\int_{0}^{t}\left\langle\int_{0}^{\tau} \dot{Y}(\tau-s) \int_{0}^{1} g_{v i}^{\prime}\left(\theta u_{x}(s)+(1-\theta) \tilde{u}_{x}(s)\right)\left(u_{x}-\tilde{u}_{x}\right) d \theta d s, w_{t x}\right\rangle d \tau\right| \\
& \leq \int_{0}^{t} \int_{0}^{\tau} C_{5} \tilde{C}_{3}^{v i}\left\|w_{x}\right\| d s\left\|w_{t x}\right\| d \tau \leq t_{1} \tilde{k}^{(-1)} \frac{1}{4 \delta} \int_{0}^{t}\|w\|_{V}^{2} d \tau+\delta t_{1} \int_{0}^{t}\left\|w_{t x}\right\|^{2} d \tau
\end{aligned}
$$

for any $\delta>0$. Hence, if we choose $\delta$ small enough, (3.64) along with (3.65)-(3.68) gives

$$
\left\|w_{t}\right\|^{2}+k^{-1}\|w(t)\|_{V}^{2} \leq L \int_{0}^{t}\|w(\tau)\|_{V}^{2} d \tau
$$

By Gronwall's inequality we obtain $w(t) \equiv 0$ on $[0, t]$, which shows that the weak solution is unique on $\left[0, t_{1}\right]$.

Now we can show that $u^{(1)}$ possesses extra smoothness, i.e., $u^{(1)} \in C\left(0, t_{1} ; V\right)$ and $u_{t}^{(1)} \in$ $C\left(0, t_{1} ; H\right)$. We consider the systems

$$
\begin{aligned}
& w_{t t}+w_{x x}+\varepsilon w_{x x t}=F-\frac{\partial}{\partial x}\left(g_{e}\left(u_{x}^{(1)}\right)+Y(0) g_{v}\left(u_{x}^{(1)}, \dot{u}_{x}^{(1)}\right)\right) \\
& +\frac{\partial}{\partial x}\left(\int_{t-r}^{t} \dot{Y}(t-s) g_{v}\left(u_{x}^{(1)}, \dot{u}_{x}^{(1)}\right) d s\right) \\
& w(0, x)=0 \\
& w_{t}(0, x)=0
\end{aligned}
$$

and

$$
\begin{aligned}
& v_{t t}+v_{x x}+\varepsilon w_{x x t}=0 \\
& v(0, x)=\varphi_{1} \\
& v_{t}(0, x)=0
\end{aligned}
$$


Since $\frac{\partial}{\partial x}\left(g_{e}\left(u_{x}^{(1)}\right)+Y(0) g_{v}\left(u_{x}^{(1)}, \dot{u}_{x}^{(1)}\right)+\int_{t-r}^{t} \dot{Y}(t-s) g_{v}\left(u_{x}^{(1)}, \dot{u}_{x}^{(1)}\right) d s\right) \in L^{2}\left(0, t_{1} ; V^{*}\right)$ by [7] we get that a unique weak solution $w$ and $v$ of $(3.72)$ and (3.75), respectively, exists on $\left[0, t_{1}\right]$ with $w, v \in C\left(0, t_{1} ; V\right)$ and $w_{t}, v_{t} \in C\left(0, t_{1} ; H\right)$. However, $w+v$ and $u^{(1)}$ both solve (3.24), so by uniqueness $w+v=u^{(1)}$ and $u^{(1)} \in C\left(0, t_{1} ; V\right), u_{t}^{(1)} \in C\left(0, t_{1} ; H\right)$.

\section{Continuation of the weak solution}

We suppose that a unique weak solution $u^{(K)}$ exists on $\left[0, t_{K}\right]$ with $u^{(K)} \in C\left(0, t_{K} ; V\right), \quad u_{t}^{(K)} \in$ $C\left(0, t_{K} ; H\right)$, and we consider the next time interval, $\left[t_{K}, t_{K+1}\right]$. (We note that the jump points $t_{j}$ are defined by $\dot{u}\left(t_{j}\right)=0$, hence continuity of $u_{t}$ across these points is guaranteed.) We have the following system:

$$
\begin{aligned}
& u_{t t}-\varepsilon u_{x x t}-\frac{\partial}{\partial x}\left(u_{x}+g_{e}\left(u_{x}\right)+Y(0) g_{v}\left(u_{x}, \dot{u}_{x}\right)+\int_{t-r}^{t} \dot{Y}(t-s) g_{v}\left(u_{x}(s)\right) d s\right. \\
& \left.+\sum_{k=1}^{K}(-1)^{k+1} Y\left(t-t_{k}\right)\left[g_{v i}\left(u_{x}^{(K)}\left(t_{k}\right)\right)-g_{v d}\left(u_{x}^{(K)}\left(t_{k}\right)\right)\right]\right)=F(t) \quad \text { in } V^{*} \\
& u(t, 0)=0 \\
& u\left(t_{1}, x\right)=\tilde{\varphi}_{0} \\
& u_{t}(0, x)=0 \\
& u(t, x)=\tilde{\varphi}_{1}, \quad t \leq t_{K},
\end{aligned}
$$

where $\tilde{\varphi}_{0}=u^{(K)}\left(t_{K}\right)$ and

$$
\tilde{\varphi}_{1}=\left\{\begin{array}{ll}
\varphi_{1} & \text { if } t<0 \\
u^{(K)} & \text { if } 0<t<t_{K} .
\end{array}\right\}
$$

We can use the same method as before to show that a unique weak solution exists on $\left[t_{K}, t_{K+1}\right]$ : first we give an apriori estimate, then introduce Galerkin approximations and show that they converge to a weak solution. If we examine this system we can see that we can perform the same estimates as before (except that now we integrate from $t_{K}$ to $t$, where $\left.t_{K} \leq t \leq t_{K+1}\right)$. There are only two terms that are different from those in the previous calculations, the extra jump term and the history integral. To estimate the jump term we have:

$$
\begin{aligned}
& \left|\int_{t_{K}}^{t}\left\langle(-1)^{K+1} Y\left(\tau-t_{K}\right)\left[g_{v i}\left(u_{x}^{(K)}\left(t_{K}\right)\right)-g_{v d}\left(u_{x}^{(K)}\left(t_{K}\right)\right)\right], u_{t x}\right\rangle d \tau\right| \\
& \leq \int_{t_{K}}^{t}(-1)^{K+1} Y\left(\tau-t_{1}\right)\left\|g_{v i}\left(u_{x}^{(K)}\left(t_{K}\right)\right)-g_{v d}\left(u_{x}^{(K)}\left(t_{K}\right)\right)\right\|\left\|u_{t x}\right\| d \tau
\end{aligned}
$$




$$
\leq C_{4}\left\|g_{v i}\left(u_{x}^{(K)}\left(t_{K}\right)\right)-g_{v d}\left(u_{x}^{(K)}\left(t_{K}\right)\right)\right\| \int_{t_{K}}^{t}\left\|u_{t x}\right\| d \tau \leq \tilde{C} \int_{t_{K}}^{t}\left\|u_{t x}\right\|^{2} d \tau
$$

using the fact that $u^{(K)} \in C\left(0, t_{K} ; V\right)$ and the assumption A3). The history integral can be split into three terms:

$$
\begin{aligned}
& \int_{t-r}^{t} \dot{Y}(t-s) g_{v}\left(u_{x}, \dot{u}_{x}\right) d s=\int_{t-r}^{0} \dot{Y}(t-s) g_{v}\left(\varphi_{1 x}(s)\right) d s+\int_{0}^{t_{K}} \dot{Y}(t-s) g_{v}\left(u_{x}^{(K)}, \dot{u}_{x}^{(K)}\right) d s \\
& +\int_{t_{K}}^{t} \dot{Y}(t-s) g_{v}\left(u_{x}, \dot{u}_{x}\right) d s .
\end{aligned}
$$

Again, all these terms can be estimated as before using the previous bounds on $u^{(K)}$. Thus we obtain

$$
\left\|u_{t}(t)\right\|^{2}+\tilde{\nu}\|u(t)\|_{V}^{2}+\theta \int_{t_{K}}^{t}\left\|u_{t}\right\|_{V}^{2} d \tau \leq \tilde{C}\left(\varphi_{0}, \varphi_{1}, \tilde{\varphi}_{0}, \tilde{\varphi}_{1}, F, t_{K+1}\right)
$$

Galerkin approximations can be introduced as before and we can establish the same kind of convergences as $(3.45)-(3.49)$ now in the interval $\left[t_{K}, t_{K+1}\right]$. The analogue of Lemma 3.1 is straightforward and the limit process can be carried out as before. Thus we obtain a weak solution $\tilde{u}$ on the interval $\left[t_{K}, t_{K+1}\right]$, which is again unique. We can argue as before that it has the required smoothness and thus the proof of Theorem 2.1 is complete.

\section{$5 \quad$ Numerical Results}

The model mentioned in the introduction was tested on a series of physical experiments with filled rubber samples. Here we report on one type of dynamic experiment that was performed with a lightly filled rubber sample. Free release experiments were conducted with the rubber rod having a $3 \mathrm{lb}$ tip mass at one end (the other end was fixed). Initially the sample was lifted, so that no compression or extension occurred, then the support was removed and the mass fell freely. (More details on the experiments can be found in [10].) To model this particular sample we use a cubic polynomial for $g_{e}(\epsilon)=a_{1} \epsilon+a_{2} \epsilon^{2}+a_{3} \epsilon^{3}$, and we suppose that $g_{v}$ is linear and does not depend on $\dot{\epsilon}$, i.e., $g_{v}=g_{v i}=g_{v d}$. Thus we use the following model:

$$
\begin{aligned}
& \rho A_{c} \frac{\partial^{2} u}{\partial t^{2}}=\frac{\partial}{\partial x}\left[A_{c} g_{e}\left(\frac{\partial u}{\partial x}\right)+A_{c} Y(0) g_{v}\left(\frac{\partial u}{\partial x}\right)+A_{c} \int_{t-r}^{t} \dot{Y}(t-s) g_{v}\left(\frac{\partial u}{\partial x}\right) d s\right] \\
& \text { for } 0<x<\ell \\
& \left.A_{c}\left[g_{e}\left(\frac{\partial u}{\partial x}\right)+A_{c} Y(0) g_{v}\left(\frac{\partial u}{\partial x}\right)+A_{c} \int_{t-r}^{t} \dot{Y}(t-s) g_{v}\left(\frac{\partial u}{\partial x}\right) d s\right]\right|_{x=\ell}=f(t)
\end{aligned}
$$




$$
\begin{aligned}
& u(t, 0)=0 \\
& u(0, x)=u_{t}(0, x)=0 \\
& u(t, x)=0, \quad t<0,
\end{aligned}
$$

where $Y(\tau)=c_{2} e^{c_{1} \tau}$. Using the load cell data from the experiment, we set up a parameter identification problem to find the parameters: $\rho, a_{1}, a_{2}, a_{3}, c_{1}, c_{2}$. (Note that in our model (5.84)-(5.88) we did not include Kelvin-Voigt type damping, while it was important for the theoretical result. We can think of its coefficient $\varepsilon \approx 0$, and thus reconcile the theoretical and numerical results.) For spatial discretization we used linear splines, while piecewise constant elements were used in the time discretization. (More details on the computational technique for dealing with the integral term can be found in [3, 4].) We used Matlab optimization routines for the inverse problem. The computed result shows very good agreement with the collected data (Figure 2).

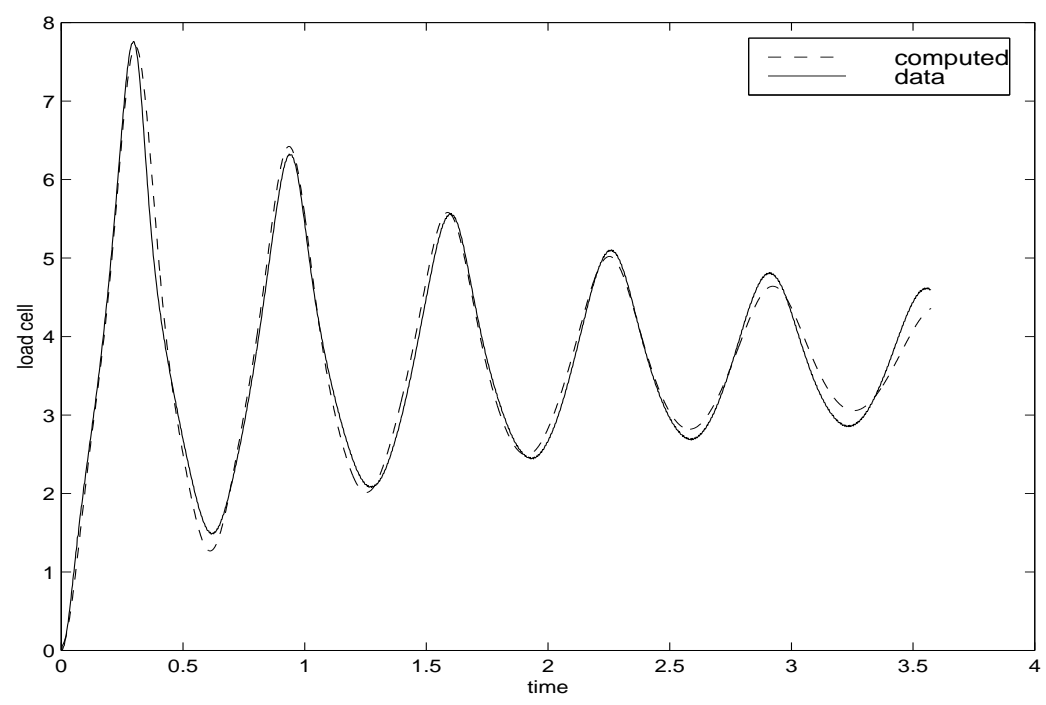

Figure 2: $3 \mathrm{lb}$ extra weight, relative error $3 \%$

This agreement is maintained across different kinds of experiments (quasi-static as well as dynamic) which provides evidence that this type of model works well in approximating the behavior of filled rubber samples. A paper on our detailed modeling and computational results for quasi-static and dynamic experiments is currently under preparation.

Acknowledgment: The experiments discussed above were carried out at Lord Corporation and the authors are grateful to Lynn Yanyo, Mike Gaitens, Nancy Lybeck and Yue Zhang 
for their substantial collaborations on experimental components of the elastomer modeling project which motivated the theoretical efforts reported on in this paper.

\section{References}

[1] J.P. Aubin, Un théorème de compacité, C.R. Acad. Sci. Vol. 256 (1963), 5042-5044.

[2] H. Bellout, F. Bloom and J. Necas, Existence of global weak solutions to the dynamical problem for a three-dimensional elastic body with singular memory, SIAM J. Math. Anal. Vol. 24 (1993), 36-45.

[3] H. T. Banks, R. H. Fabiano and Y. Wang, Estimation of Boltzmann damping coeffcients in beam models, LCDS/CCS Tech. Rep. 88-13 Brown University, July, 1988; "COMCON Conf. on Stabilization of Flexible Structures" (Montpellier, France, Dec. 1987), Opt. Software, Inc., New York, 1988, 13-35.

[4] H. T. Banks, R. H. Fabiano and Y. Wang, Inverse problem techniques for beams with tip body and time hysteresis damping, Matemática Aplicada e Computacional Vol. 8 (1989), 101-118.

[5] H.T.Banks, D.S. Gilliam and V.I. Shubov, Well-posedness for a one dimensional nonlinear beam, CRSC-TR94-18, NCSU, October, 1994; "Computation and Control IV," Birkhäuser, Boston, 1995, 1-21.

[6] H.T.Banks, D.S. Gilliam and V.I. Shubov, Global solvability for damped nonlinear hyperbolic systems, CRSC-TR95-25, NCSU, August, 1995; Differential and Integral Equations Vol. 10 (1997), 309-332.

[7] H. T. Banks, K. Ito and Y. Wang, Well-posedness for damped second order systems with unbounded input operators, CRSC-TR93-10, NCSU, June 1993; Differential and Integral Equations Vol. 8 (1995), 587-606.

[8] H. T. Banks and N. J. Lybeck, Modeling methodology for elastomer dynamics, CRSCTR96-29, NCSU, Sept. 1996; "Systems and Control in the Twenty-First Century," (C. Byrnes, et. al., eds.), Birkhäuser, Boston, 1996, 37-50.

[9] H.T. Banks, N. Lybeck, B. Munoz and L. Yanyo, Nonlinear elastomers: modeling and estimation, CRSC-TR96-26, NCSU, June, 1995; Proc. 3rd IEEE Mediterranean Symp. on New Directions in Control and Automation, July, 1995, Vol. 1, 1-7. 
[10] H.T. Banks, N. Lybeck, M. J. Gaitens, B. C. Muñoz and L.C. Yanyo, Modeling the dynamic mechanical behavior of elastomers, CRSC-TR95-19, NCSU, June, 1995.

[11] H.T. Banks, N. Lybeck, M. J. Gaitens, B. C. Muñoz, L.C. Yanyo, Computational methods for estimation in the modeling of nonlinear elastomers, CRSC-TR95-40, NCSU, Dec.,1995; Kybernetika Vol. 32 (1996), 526-542.

[12] H. Bellout and J. Necas, Existence of global weak solutions for a class of quasilinear integro-differential equations describing visco-elastic materials, Math. Ann., Vol. 299 (1994), 275-291.

[13] H.T. Banks, G.A. Pinter, L.K. Potter, M. J. Gaitens and L.C. Yanyo, Modeling of nonlinear hysteresis in elastomers, to appear.

[14] H.T. Banks, G.A. Pinter, L.K. Potter, B. C. Muñoz and L.C. Yanyo, Estimation and control related issues in smart material structures and fluids, CRSC-TR98-2, NCSU, Jan.,1998; " $4^{\text {th }}$ International Conference on Optimization Techniques and Applications," Perth, Australia, 1998, 19-34.

[15] B.D. Coleman and M.E. Gurtin, Waves in materials with memory II. On the growth and decay of one-dimensional acceleration waves, Arch. Rat. Mech. Anal. Vol. 19 (1965), 239-265.

[16] M. Doi and S.F. Edwards , Dynamics of concentrated polymer systems, J. Chem. Soc. Faraday Vol. 74 (1978), 1789-1832; Vol. 75 (1979), 38-54.

[17] C.M. Dafermos and J.A. Nohel, Energy methods for nonlinear hyperbolic Volterra integrodifferential equations, Comm. Partial Differential Equations Vol. 4 (1979), 219-278.

[18] C.M. Dafermos and J.A. Nohel, A nonlinear hyperbolic Volterra equation in viscoelasticity, "Contributions to Analysis and Geometry", John Hopkins University Press, Baltimore, Md., 1981, 87-116.

[19] H. Engler, Weak solutions of a class of quasilinear hyperbolic integro-differential equations describing viscoelastic materials, Arch. Rat. Mech. Anal. Vol. 113 (1991), $1-38$. 
[20] H. Engler, Corrigendum to "Weak solutions of a class of quasilinear hyperbolic integro-differential equations describing viscoelastic materials", Arch. Rat. Mech. Anal. Vol. 130 (1995), 401.

[21] H. Engler, Global smooth solutions for a class of parabolic integrodifferential equations, Trans. Amer. Math. Soc. Vol. 348 (1996), 267-290.

[22] A.R. Johnson, A. Tessler and M. Dambach, Dynamics of thick viscoelastic beams, J. Engr. Materials and Tech. Vol. 119 (1997), 273-278.

[23] H.M. Laun, Description of the nonlinear shear behavior of a low density polyethylene melt by means of an experimentally determined strain dependent memory function, Rheol. Acta Vol. 17 (1978), 1-15.

[24] G.A. Lesieutre, Modeling frequency-dependent longitudinal dynamic behavior of linear viscoelastic long fiber components, J. Composite Materials Vol. 28 (1994), 17701782.

[25] G.A. Lesieutre and K. Govindswamy, Finite element modeling of frequencydependent and temperature-dependent dynamic behavior of viscoelastic materials in simple shear, Int. J. Solids Structures Vol. 33 (1996), 419-432.

[26] J.L. Lions, "Quelques Méthodes de Resolution des Problémes aux Limites Non Linéaires," Dunrod/Gauthier-Villars, Paris, 1969.

[27] R.C. MacCamy, A model for one-dimensional nonlinear viscoelasticity, Q. Appl. Math. Vol. 35 (1977), 21-33.

[28] J. Milota, J. Necas and V. Sverak, On weak solutions to a viscoelasticity model, Comment. Math. Univ. Carolinae Vol. 31 (1990), 557-565.

[29] J.A. Nohel, R.C. Rogers and A.E. Tzavaras, Weak solutions for a nonlinear system in viscoelasticity, Comm. Partial Differential Equations Vol. 13 (1988), 97-127.

[30] A.W. Naylor and G.R. Sell, "Linear Operator Theory in Engineering and Science," Springer Verlag, New York, 1982.

[31] R.W. Ogden, "Nonlinear Elastic Deformations," Dover Publications, Mineola, N.Y., 1997. 
[32] P.E. Rouse, A theory of the linear viscoelastic properties of dilute solutions of coiling polymers, J. Chem. Phys. Vol. 21 (1953), 1271-1280.

[33] R.S. Rivlin, Large elastic deformations of isotropic materials I, II, III, Phil. Trans. Roy. Soc. A Vol. 240 (1948), 459-490, 491-508, 509-525.

[34] M. Renardy, W.J. Hrusa and J.A. Nohel, "Mathematical Problems in Viscoelasticity," Longman Group, Boston, London, Melbourne, 1987.

[35] O. Staffans, On a nonlinear hyperbolic Volterra equation, SIAM J. Math. Anal. Vol. 11 (1980), 793-812.

[36] B.H. Zimm, Dynamics of polymer molecules in dilute solutions: viscoelasticity, low birefringence, and dielectric loss, J. Chem. Phys. Vol. 24 (1956), 269-278. 